\title{
A Review of Diagnostic Methods and Results for HIV-associated Disseminated Histoplasmosis: Shouldn't We be Involving Pathologists More Systematically?
}

Mathieu Nacher ( $\nabla$ mathieu.nacher66@gmail.com )

Centre Hospitalier Andree Rosemon https://orcid.org/0000-0001-9397-3204

\section{Audrey Valdes}

Centre Hospitalier Andree Rosemon

\section{Antoine Adenis}

Centre Hospitalier Andree Rosemon

Philippe Abboud

centre hospitalier andree rosemon

Magalie Demar

centre hospitalier andree rosemon

\section{Felix Djossou}

Centre Hospitalier Andree Rosemon

\section{Loic Epelboin}

centre hospitalier andree rosemon

\section{Caroline Misslin}

centre hospitalier de l'ouest guyanais

\section{Denis Blanchet}

Centre Hospitalier Andree Rosemon

\section{Pierre Couppie}

Centre Hospitalier Andree Rosemon

Kinan Drak Alsibai

Centre Hospitalier Andree Rosemon

\section{Research}

Keywords: Diagnostic methods, HIV, pathologists, histoplasmosis

Posted Date: October 23rd, 2020

DOI: https://doi.org/10.21203/rs.3.rs-95731/v1 
License: (c) (i) This work is licensed under a Creative Commons Attribution 4.0 International License. Read Full License 


\section{Abstract}

Background. Disseminated histoplasmosis is a major killer of HIV-infected persons in Latin America. Antigen detection, fungal culture, or Polymerase Chain Reaction are often not available, but cytology and histology are usually present in most hospitals and may represent an important diagnostic alternative. In this study, we review 34 years of clinical experience to describe the respective place of cytology and histology to diagnose disseminated histoplasmosis.

Methods. Between January 1st, 1981 and October 1st, 2014, a retrospective multicentric study was performed on 349 patients with confirmed disseminated histoplasmosis.

Results. Whereas bone marrow was by far the most common sample taken, only $14.9 \%$ of samples were screened using cytopathology, the second most frequent sample taken was bonchoalveolar lavage for which $9.9 \%$ were subjected to cytopathological analysis, and finally spinal fluid for which $16.4 \%$ were subjected to cytopathological analysis. The samples most systematically sent to pathology were liver biopsies, lower digestive tract, and lymphnode biopsies and the most contributive in terms of positive results were lower digestive tract (72.9\% positives), lymph node (66.1\%), and liver (50.7\%). $97.2 \%$ of bone marrow samples were subjected to direct examination by the mycologist, the second most frequent sample taken was bronchoalveolar lavage for which $97 \%$ were subjected to direct examination. Positive direct examination was independently associated with death (aHR=1.5 (95\% Cl=1-2.2), and positive pathology was associated with less mortality $((\mathrm{aHR}=0.66(95 \% \mathrm{Cl}=0.44-1)$.

Conclusions. Opportunities for a rapid diagnosis were regularly missed, notably for bone marrow samples which could have been examined using complementary staining methods to those of mycologist.

\section{Introduction}

H. capsulatum is the causative agent of disseminated histoplasmosis, a major but neglected Acquired Immune Deficiency Syndrome (AIDS)-defining infection and cause of death in Latin America.[1-3] In the tissues, Histoplasma capsulatum (H. capsulatum) presents as small spherical or ovoid yeasts measuring 2 to $6 \mu \mathrm{m}$ [4]. After inhalation, it makes a dimorphic transition from must-leaven to yeast, to enter host histiocytes/macrophages, and is able to survive intracellularly. It can thus proliferate during active infection, and persist during asymptomatic infections, and then reactivate [5].

The severity of disease after inhalation of $H$. capsulatum is influenced by the intensity of exposure and the host's immunity. Hence there is a spectrum of disease severity ranging from asymptomatic infections or mild pulmonary disease in the case of low inoculums and normal immunity, whereas large inoculums may lead to severe pulmonary infections. In immunocompromised patients the infection progressively disseminates to other organs leading to a variety proteiform and life-threatening disease. Depending on which organs are affected: lungs, gastrointestinal tract, liver or lymph nodes which may all be involved in the same patient. Since the beginning of the AIDS pandemic, there has been a growing reservoir of patients susceptible to progressive disseminated histoplasmosis, which given the difficulties of diagnosis 
and the lack of awareness have resulted in a staggering burden of fatalities [6]. Histoplasmosis has in fact often been ranked as the most common AIDS-defining infection and cause of death for patients with Cluster of Differentiation-4 (CD4) cell counts $<200$ cells $/ \mathrm{mm}^{3}[7,8]$.

French Guiana is endemic for histoplasmosis and there is a high prevalence of HIV infection[9]. The incidence of disseminated histoplasmosis in HIV patients is estimated to be 1.5 per 100 persons-years overall, and greater than 10 per 100 persons-years in patients with CD4 counts under 50/mm3[7, 10]. In South America, histoplasmosis is also frequent [11].

Before the advent of the AIDS epidemic, animal samples [12]or autopsy specimens were often used to study Histoplasmosis [13]. In the absence of availability of antigen detection testing outside of the USA, the reference methods to study histoplasmosis in immunocompromised patients are cytological and histological examination and fungal culture with a sensitivity of $65 \%$ and $80 \%$, respectively [14-16]. Molecular detection by polymerase chain reaction (PCR), and serological detection in plasma are also used. However, the sensitivity of histoplasmosis detection methods is highly variable, and may differ geographically depending on the experience and the methods used in each center [14, 17-19]. Moreover, there may be differences according to the type of organ sample or the immune status of the patient [20, 21]. In endemic countries, antigen detection, fungal culture, or Polymerase Chain Reaction (PCR) are often not available, but cytology and histology are usually present in most hospitals and may represent an important diagnostic alternative. In this study, we review 34 years of clinical experience to describe the respective place of cytology and histology to diagnose disseminated histoplasmosis.

\section{Methods}

\section{Study design}

Between January 1st, 1981 and October 1st, 2014, a retrospective multicentric study was performed on patients with confirmed disseminated histoplasmosis.

\section{Study population}

Co-infections with Human Immunodeficiency Virus (HIV) and histoplasmosis were included in the HIV and Histoplasmosis database of French Guiana. The inclusion criteria were confirmed HIV infection; age $>18$ years; and first proven episode of histoplasmosis (European Organization for Research and Treatment of Cancer / Mycoses Study Group (EORTC/MSG) criteria[22]);.

\section{Study conduct}

This database included incident cases of HIV-associated histoplasmosis in the three hospitals of the 3 main cities of French Guiana. Clinical, biological, immunovirological and therapeutic data were collected on a standardized case record form until October 2014. Hospitalized cases of incident HIV-associated histoplasmosis were included. We collected sex, age, country of birth, symptoms on admission, clinical 
entrance examination, treatment received, and survival data during the study period, immunovirological status, standard biological examinations, medical imaging, endoscopies and procedures performed to identify H. capsulatum, mycology, cytology and pathology.

\section{Statistical analysis}

STATA $^{\odot}$ software (College Station, Texas, USA) was used. Quantitative variables were described with medians and interquartile ranges, they were compared between groups using ranksum non-parametric tests where appropriate. For qualitative variables, Chi2 or Fisher tests were computed comparing the proportions between groups. Kaplan Meier curves were computed and Cox proportional hazards modelling was used to adjust for potential confounding. Statistical significance was set at $5 \%$.

\section{Ethical and regulatory aspects}

The research was approved by the by the the French National Institute of Health and Medical Research institutional review board (Comité d'Evaluation Ethique de l'Institut National pour la Santé Et la Recherche Médicale (CEEI INSERM) (IRB0000388, FWA00005831 18/05/2010), the Comité Consultatif pour le Traitement de l'Information pour la Recherche en Santé(CCTIRS) (N 10.175bis, 10/06/2010), and the Commission Nationale Informatique et Libertés (CNIL) ( ${ }^{\circ}$ JZU0061856X, 07/16/2010).

\section{Results}

There were 349 observations of HIV-associated disseminated histoplasmosis in the database. The average age was 40 years ( \pm 9.7 years). The sex ratio was 1.9 male per female. The median number of sites from which samples were taken to try to identify the pathogen was 2 but varied (Interquartile range $(\mathrm{IQR})=1-3$, maximum 5). Figure 1 shows the "cascade" from site sample to diagnosis for cytopathology. Whereas bone marrow was by far the most common sample taken, only $14.9 \%$ of samples were screened using cytopathology, the second most frequent sample taken was bonchoalveolar lavage for which $9.9 \%$ were subjected to cytopathological analysis, and finally spinal fluid for which $16.4 \%$ were subjected to cytopathological analysis. The samples most systematically sent to pathology were liver biopsies, lower digestive tract, and lymphnode biopsies and the most contributive in terms of positive results were lower digestive tract ( $72.9 \%$ positives), lymph node (66.1\%), and liver $(50.7 \%)$.

Whereas those with positive histopathology were less likely to have a positive culture $(O R=0.12,95 \% \mathrm{Cl}=$ $0.05-0.26, \mathrm{P}<0.0001)$ they were more likely to have a positive direct examination $(\mathrm{OR}=1.8,95 \% \mathrm{Cl}=$ $1.13-2.83, \mathrm{P}=0.008)$. Only $10 / 349(2.9 \%)$ were diagnosed with direct examination alone (only positive result), 29/349 (8.3\%) were diagnosed by pathology alone (only positive result), and 100/349 (28.65\%) by culture alone (only positive result). Eighty-nine persons (25.5\%) had both a positive direct examination and a positive culture, $18 / 349(5.1 \%)$ had both a positive direct examination and positive pathology, and 25/349 (7.1\%) had both a positive pathology and a positive culture. Overall 139 patients were only diagnosed with a single technique, 132 by 2 , and $77(22 \%)$ by direct examination, pathology, and culture. The patients more likely to have 2 or 3 positive techniques were those with cutaneous and oral lesions. 
Persons with respiratory presentations were less likely to have a positive diagnosis by pathology than those without a respiratory presentation $(35.5 \%$ vs $49.5 \%, P=0.009)$. By contrast, those with cutaneous signs were significantly more likely to have a positive pathology diagnosis $(79.2 \%$ vs $40.1 \%, P<0.0001)$. Similarly, those with oral lesions were significantly more likely to have a positive pathology diagnosis (78.9\% vs $40.7 \%, P=0.001)$. Patients with Superficial lymphadenopathies $<2 \mathrm{~cm}$ were significantly less likely to have a positive pathology diagnosis than those without ( $37.9 \%$ vs $56.7 \%, P=0.019$ ), while those with lymphadenopathies $>2 \mathrm{~cm}$ were more likely to have a positive diagnosis by pathology $(55.5 \% \mathrm{vs}$ $38.1 \%, P=0.028$ ). Patients having benefitted from a lower digestive tract (but not the upper tract) endoscopy were significantly more likely to have a positive pathology diagnosis than those who did not (59.5\% vs $31.2 \%, P=0.002$ ). Patients having benefitted from a abdominopelvic CT scan (but not thoracic) were significantly more likely to have a positive pathology diagnosis than those who did not ( $52 \%$ vs $39.3 \%, P=0.03)$.

Patients with a positive pathology examination had significantly higher platelet count $(171,000$ per mm3 $(\mathrm{IQR}=39,800-278,000))$ than those who did not $(124,000$ per mm3 $(\mathrm{IQR}=38,000-219,000)), \mathrm{P}=0.03$. Patients with a positive pathology examination had significantly lower TGO concentrations (45 IU (IQR = $30-73))$ than those who did not (64 IU (IQR = 35-112)), $\mathrm{P}=0.0007$. Patients with a positive pathology examination had significantly lower LDH concentrations (374 IU (IQR = 252-722)) than those who did not (456 IU (IQR = 302-1132)), P = 0.01. Whereas those diagnosed by culture or direct examination did not have any differences in diagnostic delay, those diagnosed by pathology seemed to have a shorter diagnostic delay (50days (IQR $=22-225)$ vs $91(\mathrm{IQR}=29-237), \mathrm{P}=0.05$.

Figure 2 shows the "cascade" from sample to diagnosis for direct examination. Bone marrow was by far the most common sample taken, and $97.2 \%$ of samples were subjected to direct examination by the mycologist, the second most frequent sample taken was bronchoalveolar lavage for which $97 \%$ were subjected to direct examination, and finally liver biopsy for which $83.3 \%$ were subjected to cytopathological analysis. The most contributive in terms of the proportion of positive results for direct examination were cutaneomucous ( $73.7 \%$ positives, fourth in frequency), lower digestive $(52.5 \%$ positives, third in frequency), bronchoalveolar lavage (46.9\% positives, second in frequency), and bone marrow (34.6\% positives, but first in frequency).

Figure 3 shows the "cascade" from sample to diagnosis for fungal culture. Bone marrow was by far the most common sample taken, and $93.9 \%$ of samples were cultured for fungi, the second most frequent sample taken was bronchoalveolar lavage for which $90.9 \%$ were cultured for fungi, and finally liver biopsy for which $70.9 \%$ were cultured for fungi. The most contributive in terms of the proportion of positive results for fungal culture were liver (86.9\%, second in frequency), lymphnodes $(79.6 \%$ positives, third in frequency), bone marrow ( $77.6 \%$ positives, and first in frequency), and lower digestive ( $74 \%$ positives, fourth in frequency). Figure 4 shows a summary of the proportion of samples analyzed and the proportion of positives. Hence, overall, less than half of samples benefitted from pathological examinations, although over half of samples examined by the pathologist were positive. 
Figure 5 shows the survival curves within the first year. Patients with a positive direct examination were more likely to die than those without. In contrast those having positive pathology seemed less likely to die than those having negative pathology, and those having a positive culture also seemed less likely to die within the next 12 months. A cox model adjusting all 3 methods of diagnosis for CD4 count, diagnostic delay, the time period, and antifungal treatment showed that a positive direct examination was significantly associated with death (adjusted hazard ratio $=1.5(95 \% \mathrm{Cl}=1-2.2), \mathrm{P}=0.048$, and positive pathology was associated with less mortality ((adjusted hazard ratio $=0.66(95 \% \mathrm{Cl}=0.44-1), \mathrm{P}=0.05$. there was no significant association for culture in the same model.

\section{Discussion}

The present results show that, for HIV patients with disseminated histoplasmosis, between direct examination, culture and pathology, pathology was the least frequently prescribed diagnostic method (45\% of patients) despite its potentially valuable yield (54.8\% positivity). This underuse of pathology was noted for non-tissular liquid samples, notably bone marrow, which was the most frequently collected sample and the most frequently positive sample. Fluid samples are usually sent to the laboratory for fungal diagnosis, where direct examination is performed on May-Günwald Giemsa-stained samples at 1000 magnification using immersion oil. For bone marrow aspiration, a variable number of smears, of sometimes heterogenous quality, are prepared for examination by cytologists, parasitologists and mycologists, and rarely by pathologists. The small fraction (14.8\%) of bone marrow samples that actually reach the pathologist may result from the lack of a sufficient number of satisfactory smears to provide to all specialties reading the slides. Another likely explanation is clinical habit, and the perception that pathologists only deal with solid tissue samples. If this is the case, then perhaps there are lost opportunities for diagnosis because pathologists apply different staining techniques that mycologists, which could have rapidly diagnosed a number of additional cases. Patients having benefitted from a positive pathological diagnosis had a shorter diagnostic delay and seemed more likely to survive that patients who did not have a positive pathology. They had higher platelet counts, lower Glutamyloxaloacetate transferase (TGO) and Lactico DesHydrogenase (LDH) concentrations than patients who had negative pathology. The interpretation of these findings could first be that diagnostic delay is shorter with pathology (within days) than with culture (weeks). Another potential explanation could be that among patients with some remaining defense against the fungal pathogen, this defense translates into tissular lesions such as granuloma which are easier to find that anergic forms were the sideration of immunity leads to very few modifications of tissues and cells around the fungus.

Direct examination was linked with death, a somewhat predictable result reflecting a greater fungal burden and hence more severity.

While some samples were rarely forwarded to pathology, others, usually solid tissues, were very frequently submitted to pathological analysis. Hence, those with cutaneous signs, those with oral lesions, those with lymphadenopathies $>2 \mathrm{~cm}$ were significantly more likely to have a positive pathology 
diagnosis. Patients having benefitted from a lower digestive tract endoscopy were also significantly more likely to have a positive pathology diagnosis than those who did not.

The present study reviewed practices and results regarding diagnosis over a 34-year period. There was a great heterogeneity between type of organ sample and the propensity to seek pathological analysis.

Given that, in the absence of antigen detection tests, pathology yields relatively rapid results, it seemed that some opportunities were regularly missed, notably for bone marrow samples which could have been examined using complementary staining methods to those of mycologists, that may have increased the proportion of direct examination positives, only at $1 / 3$ of samples examined, by adding a complementary technique to the techniques used by mycologists.

\section{Abbreviations}

AIDS: Acquired Immune Deficiency Syndrome

CEEl: Comité d'Evaluation Ethique de l'Institut National pour la Santé Et la Recherche Médicale EORTC/MSG: European Organization for Research and Treatment of Cancer / Mycoses Study Group

HIV: Human Immunodeficiency Virus

IQR: Interquartile range

LDH: Lactico Deshydrogenase

PCR: Polymerase Chain Reaction

TGO: Glutamyl-oxaloacetate transferase

\section{Declarations}

\section{Ethics approval and consent to participate}

The research was approved by the by the the French National Institute of Health and Medical Research institutional review board (CEEI INSERM) (IRB0000388, FWA00005831 18/05/2010), the Comité Consultatif pour le Traitement de l'Information pour la Recherche en Santé(CCTIRS) ( $N^{\circ} 10.175 \mathrm{bis}$, 10/06/2010), and the Commission Nationale Informatique et Libertés (CNIL) ( ${ }^{\circ} \mathrm{JZU0061856X}$, 07/16/2010).

\section{Consent for publication}

Yes.

\section{Competing interests}


No competing interests declared.

\section{Availability of data and materials}

Data may be made available upon reasonable request and clearance by the Commission Nationale Informatique et Libertés (CNIL), 3 pl Fontenoy, 75334 PARIS CEDEX 07

\section{Competing interests}

None declared

\section{Funding}

None declared

\section{Author Contributions}

“Conceptualization, MN.; methodology, MN.; formal analysis, MN.; investigation, $\mathrm{LE}, \mathrm{RB}, \mathrm{AV}, \mathrm{AA}, \mathrm{PA}, \mathrm{FD}, \mathrm{MD}, \mathrm{CM}, \mathrm{BN}, \mathrm{PC}, \mathrm{KDA}$; data curation $\mathrm{AA}, \mathrm{PC}$.; writing-original draft preparation, $\mathrm{MN}$.; review and editing, $L E, R B, A V, A A, P A, F D, M D, C M, B N, P C, K D A$.; All authors have read and agreed to the published version of the manuscript.",

\section{Acknowledgements}

To all physicians and technicians that have been involved in collecting data over the years.

\section{References}

1. Nacher M, Adenis A, Abboud P, Djossou F, Demar M, Epelboin L, et al. HIV patients dying on antituberculosis treatment: are undiagnosed infections still a problem in French Guiana? BMC Research Notes. BioMed Central; 2020;13:1-5.

2. Nacher M, Adenis A, Mc Donald S, Do Socorro Mendonca Gomes M, Singh S, Lopes Lima I, et al. Disseminated Histoplasmosis in HIV-Infected Patients in South America: A Neglected Killer Continues on Its Rampage. PLoS Negl Trop Dis. 7:e2319.

3. Adenis AA, Valdes A, Cropet C, McCotter OZ, Derado G, Couppie P, et al. Burden of HIV-associated histoplasmosis compared with tuberculosis in Latin America: a modelling study. Lancet Infect Dis. 2018;18:1150-9.

4. FN S. Schwartz J, Lahey ME, Carson RP. Histoplasmosis. Am J Med. 1955;19:410.

5. Woods JP, Heinecke EL, Luecke JW, Maldonado E, Ng JZ, Retallack DM, et al. Pathogenesis of Histoplasma capsulatum. Seminars in respiratory infections. 2001. p. 91.

6. Nacher M. Histoplasmosis in Persons Living with HIV. Multidisciplinary Digital Publishing Institute; 2020. 
7. Nacher M, Adenis A, Adriouch L, Dufour J, Papot E, Hanf M, et al. What is AIDS in the Amazon and the Guianas? Establishing the burden of disseminated histoplasmosis. Am J Trop Med Hyg. 2011;84:239-40.

8. Nacher M, Adenis A, Guarmit B, Lucarelli A, Blanchet D, Demar M, et al. What is AIDS in the Amazon and the Guianas in the 90-90-90 era? bioRxiv. Cold Spring Harbor Laboratory; 2020;

9. Modeling of the HIV epidemic and continuum of care in French Guiana [Internet]. [cited 2020 Feb 29]. Available from: https://journals.plos.org/plosone/article?id=10.1371/journal.pone.0197990

10. Nacher M, Adenis A, Blanchet D, Vantilcke V, Demar M, Basurko C, et al. Risk factors for disseminated histoplasmosis in a cohort of HIV-infected patients in French Guiana. PLoS Negl Trop Dis. 2014;8:e2638.

11. Couppié P, Herceg K, Bourne-Watrin M, Thomas V, Blanchet D, Alsibai KD, et al. The Broad Clinical Spectrum of Disseminated Histoplasmosis in HIV-Infected Patients: A 30 Years' Experience in French Guiana. Journal of Fungi. Multidisciplinary Digital Publishing Institute; 2019;5:115.

12. Emmons CW, Ashburn LL. Histoplasmosis in wild rats: Occurrence and histopathology. Public Health Reports (1896-1970). JSTOR; 1948;1416-1422.

13. Queiroz AC, Siqueira LA. Histoplasmose em material de autópsia. Revista de patologia Tropical. 1975;4:107-114.

14. Wheat LJ. Current diagnosis of histoplasmosis. Trends in microbiology. Elsevier; 2003;11:488-494.

15. Wheat LJ. Improvements in diagnosis of histoplasmosis. Expert opinion on biological therapy. Taylor \& Francis; 2006;6:1207-1221.

16. Nacher M, Blanchet D, Bongomin F, Chakrabarti A, Couppié P, Demar M, et al. Histoplasma capsulatum antigen detection tests as an essential diagnostic tool for patients with advanced HIV disease in low and middle income countries: A systematic review of diagnostic accuracy studies. PLoS Negl Trop Dis. 2018;12:e0006802.

17. Tobón AM, Agudelo CA, Rosero DS, Ochoa JE, De Bedout C, Zuluaga A, et al. Disseminated histoplasmosis: a comparative study between patients with acquired immunodeficiency syndrome and non-human immunodeficiency virus-infected individuals. Am J Trop Med Hyg. 2005;73:576-82.

18. Mata-Essayag S, Colella MT, Roselló A, de Capriles CH, Landaeta ME, de Salazar CP, et al. Histoplasmosis: a study of 158 cases in Venezuela, 2000-2005. Medicine. LWW; 2008;87:193-202.

19. Huber F, Nacher M, Aznar C, Pierre-Demar M, El Guedj M, Vaz T, et al. AIDS-related Histoplasma capsulatum var. capsulatum infection: 25 years experience of French Guiana: AIDS. 2008;22:104753.

20. Tobon AM, Agudelo CA, Rosero DS, Ochoa JE, De Bedout C, Zuluaga A, et al. Disseminated histoplasmosis: a comparative study between patients with acquired immunodeficiency syndrome and non-human immunodeficiency virus-infected individuals. Am J Trop Med Hyg. 2005;73:576-82.

21. Weydert JA, Van Natta TL, DeYoung BR. Comparison of fungal culture versus surgical pathology examination in the detection of Histoplasma in surgically excised pulmonary granulomas. Archives of pathology \& laboratory medicine. 2007;131:780-783. 
22. De Pauw B, Walsh TJ, Donnelly JP, Stevens DA, Edwards JE, Calandra T, et al. Revised definitions of invasive fungal disease from the European Organization for Research and Treatment of Cancer/Invasive Fungal Infections Cooperative Group and the National Institute of Allergy and Infectious Diseases Mycoses Study Group (EORTC/MSG) Consensus Group. Clin Infect Dis. 2008;46:1813-21.

\section{Figures}

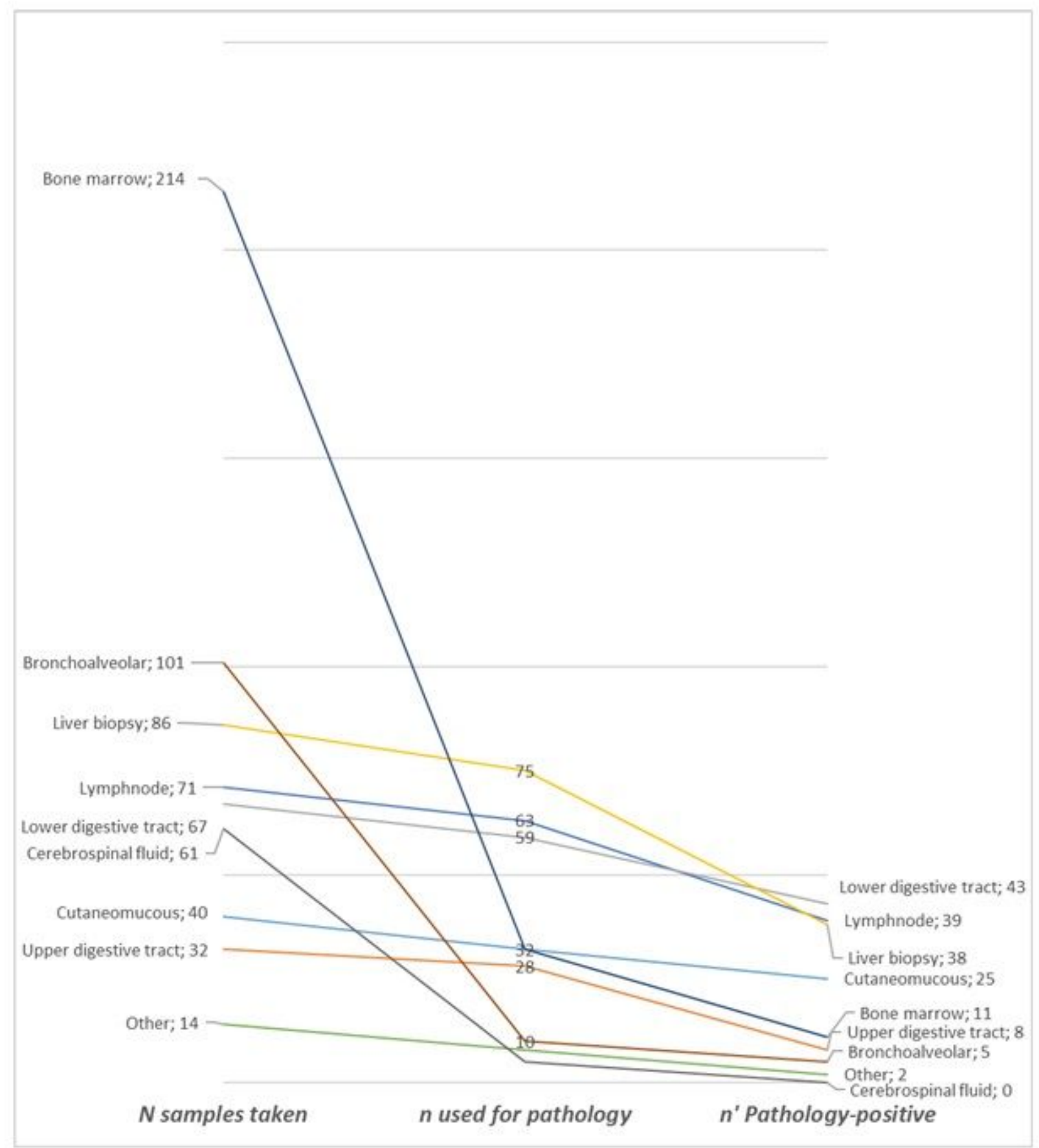

Figure 1 
Cascade from site sample collection to diagnosis for pathology.



Figure 2

Cascade from site sample collection to diagnosis for direct examination. 
Bone marrow; 214
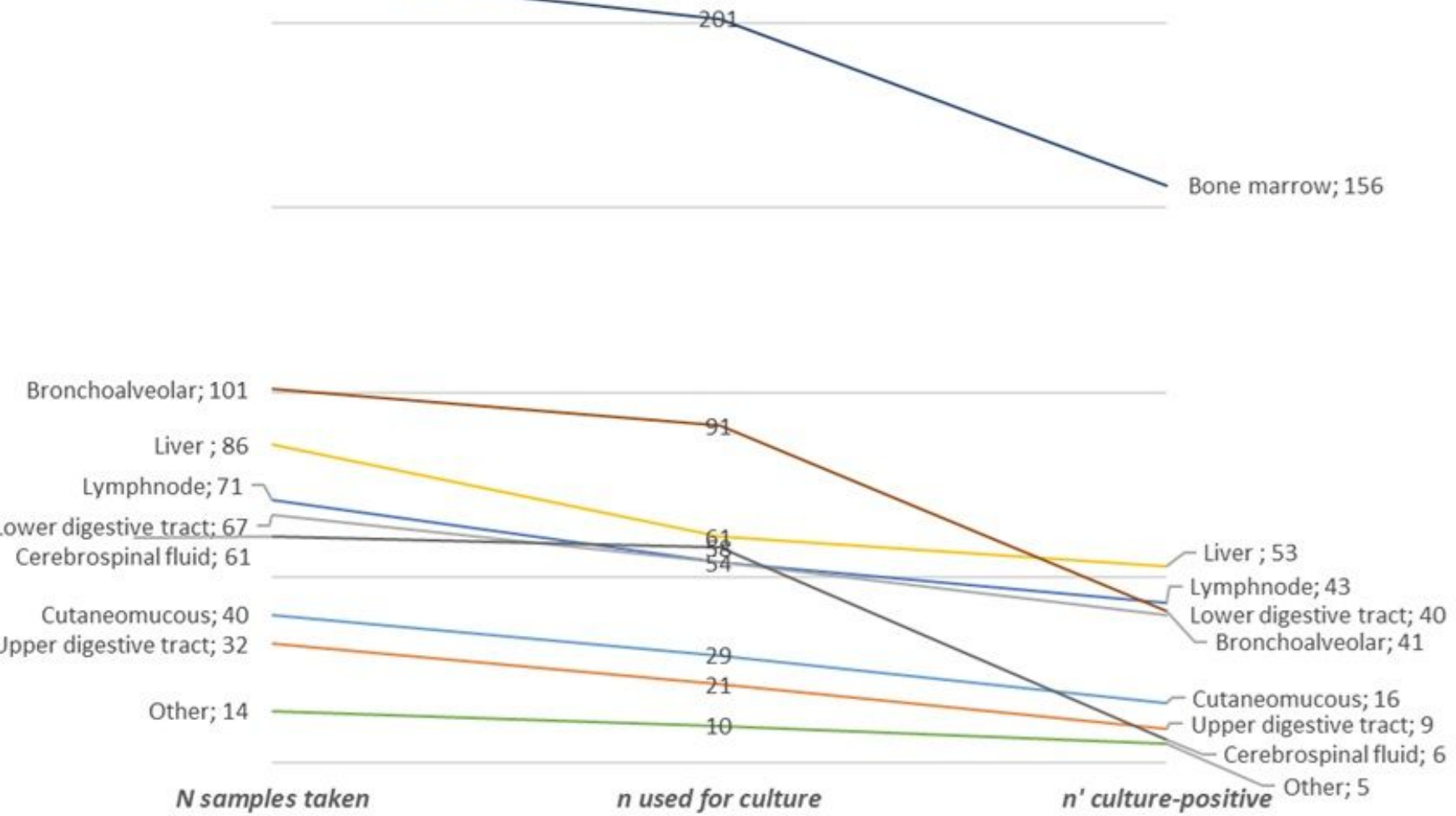

\section{Figure 3}

Cascade from site sample collection to diagnosis for fungal culture. 


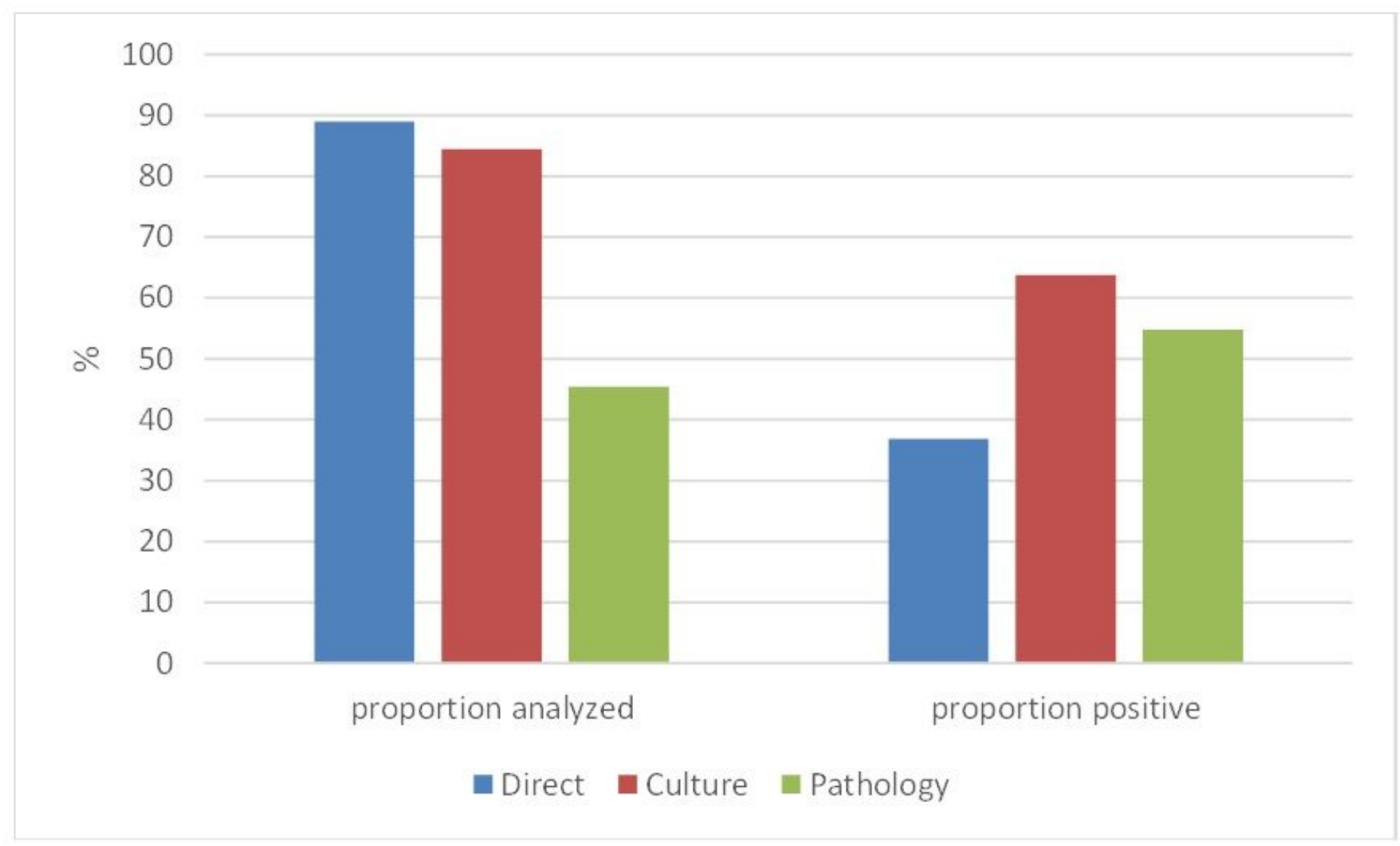

Figure 4

Overall cascade from sample to diagnosis by diagnostic method. 

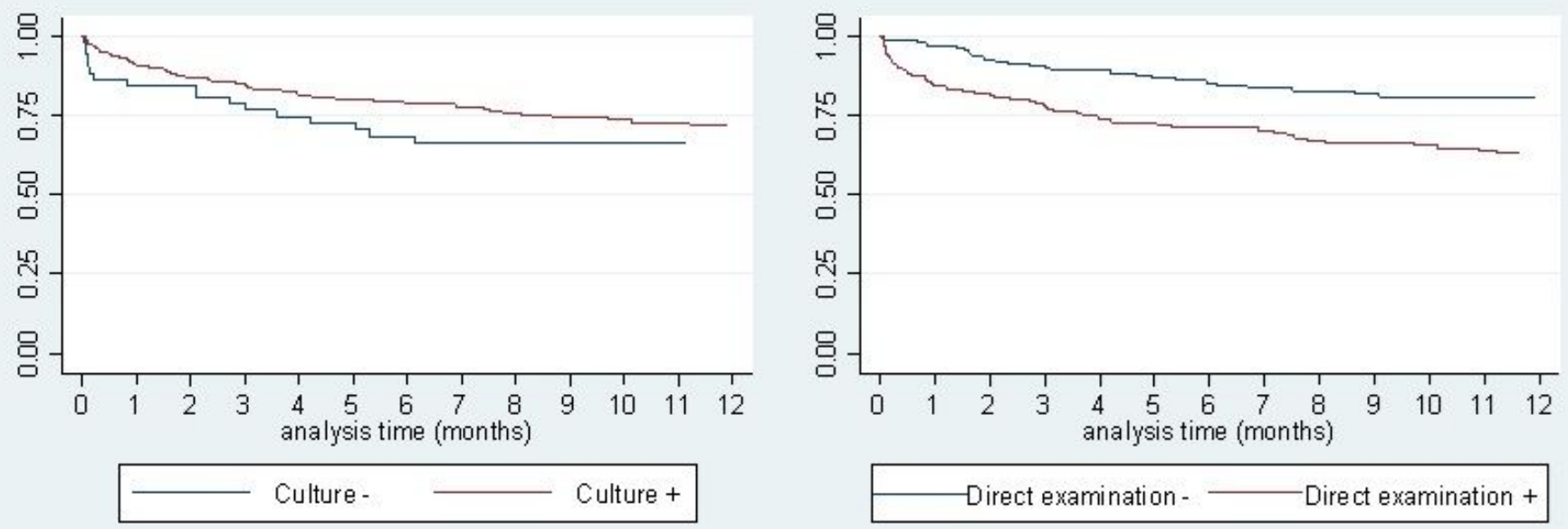

Direct examination -

Direct examination +

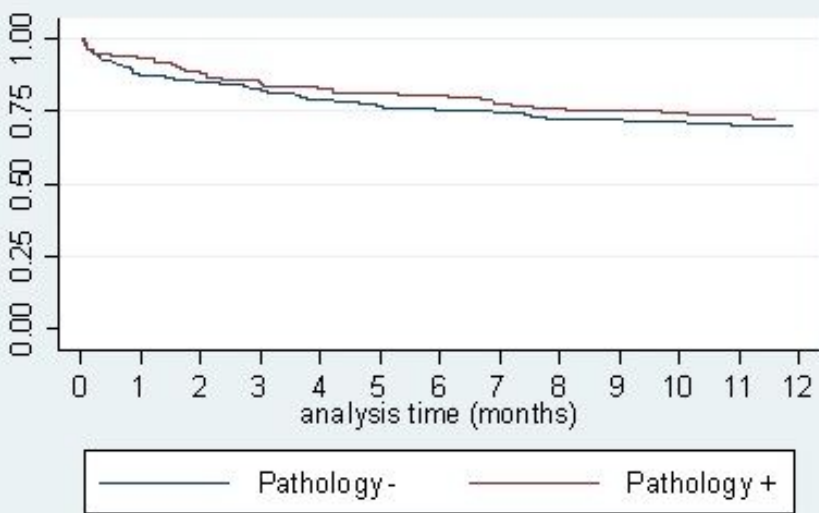

\section{Kaplan-Meier survival estimates}

\section{Figure 5}

Kaplan Meier curves for death in HIV-infected patient with disseminated histoplasmosis by diagnostic method. 